\title{
Fetal Magnetic Resonance Imaging of Choledochal Cyst
}

\author{
Dewi A Wirasasmita ${ }^{1}$, Gatot Abdurrazak ${ }^{2}$, Ahmad Yani ${ }^{3}$
}

\begin{abstract}
Choledochal cysts (CCs) are rare congenital anomaly with characterized dilatation of intra- or extrahepatic biliary duct or both. Prenatal diagnosis is increasing due to high resolution of ultrasound (US) and ultrafast sequences of magnetic resonance imaging (MRI) showing better delineation of the cyst to the adjacent structure. Considering the additional value of the fetal MRI report is still infrequent to confirm the CC case, we therefore report the type I of CC, which was previously diagnosed as a cystic biliary atresia (CBA) from US result. Postnatal MRI and histopathology prove it. Keywords: Choledochal cyst, Cystic biliary atresia, Postnatal magnetic resonance imaging, Prenatal magnetic resonance imaging. International Journal of Infertility and Fetal Medicine (2020): 10.5005/jp-journals-10016-1200
\end{abstract}

\section{INTRODUCTION}

Choledochal cysts (CC) are congenital cystic dilatation of bile ducts. The occurrence in Asia is much more common than in Western countries. Females are more common than males, with a femaleto-male ratio in the range of $3: 1-4: 1{ }^{1}$ Prenatal ultrasound (US) remains the first modality for antenatal screening. Ultrasound can suspect of CC if location at the right upper quadrant abdomen and demonstrate a connection between a dilated common bile duct and a cystic lesion. However, cystic change can be found in the obliterated biliary tract as well, so it will be difficult to distinguish the CC and cystic biliary atresia (CBA) especially if gallbladder (GB) cannot be identified by US. Fetal MRI with ultrafast sequences, large field of view, and excellent natural contrast can give additional information.

\section{Case Description}

We describe a pregnant woman gravida 2, para 1, in routine US prenatal scan it demonstrates a round cystic mass at the right upper quadrant abdomen, measuring $2.19 \times 1.85 \times 1.3 \mathrm{~mm}$, GB cannot be evaluated, suspected of a cystic biliary atresia with differential diagnosis of a CC.

Two weeks later, fetal MRI (GE HDE 1.5 T) was performed at 35 weeks' gestation for further evaluation of the cyst using T2SSFSE (TR/TE 15,000/121 ms), T2FIESTA (TR/TE 11.0/2.7 ms), and T1-2DGRE (TR/TE 120/6.7) sequences, slice thickness 4-5 mm, flip angle 80, axial, sagittal, and coronal abdominal section. The MRI revealed an extrahepatic cyst lesion, well-defined border with tapered ends at hepatic hilum (Figs $1 A$ and $B$ ), hyperintense on T2 SSFSE and T2FIESTA, isointense to the lungs on T1W (Fig. 1C). Sagittal images demonstrated the GB with normal size $(25 \times 8 \mathrm{~mm})$ and normal position (Fig. 1D), the cyst at posterior orientation and shows relationship with normal GB (Figs $1 \mathrm{E}$ and $\mathrm{F}$ ). Axial images show the cyst posterior to the duodenum. Others structure such as spleen, stomach, kidneys, small, and large bowel have normal shape and signal intensity.

A female full-term baby was born by vaginal delivery with Apgar score 9/10, weighted $3.2 \mathrm{~kg}$. The newborn was anicteric and cholic stools. The baby condition was unremarkable. Postnatal laboratory findings were normal. Follow up US was done at 24 days, 2 months, and 4 months. Ultrasound showed no enlargement of the cyst and no dilatation of intrahepatic biliary duct or sign of extrahepatic
${ }^{1}$ Department of Radiology, Premier Jatinegara Hospital, Jakarta Pusat, Jakarta, Indonesia

${ }^{2}$ Department of Obstetrics and Gynecology, Harapan Kita Women and Children National Hospital, DKI Jakarta, West Jakarta, Indonesia

${ }^{3}$ Department of Pediatric Surgery, University of Indonesia, Cipto Mangunkusumo Hospital, Jakarta Pusat, DKI Jakarta, Indonesia

Corresponding Author: Dewi A Wirasasmita, Department of Radiology, Premier Jatinegara Hospital, Jakarta Pusat, Jakarta, Indonesia, Phone: +62 811936350, e-mail: dewi_wirasasmita@yahoo.com

How to cite this article: Wirasasmita DA, Abdurrazak G, Yani A. Fetal Magnetic Resonance Imaging of Choledochal Cyst. Int J Infertil Fetal Med 2020;11(1):27-29.

Source of support: Nil

Conflict of interest: None

biliary atresia. Until 10 months old, the baby has pale stools with abdomen distended and progressively jaundice. The bilirubin direct level and liver function tests were increased. The baby was decided to undergo surgery and follow-up MRI was performed before surgery. Follow-up MRI showed dilatation of intra- and extrahepatic biliary duct and the cyst growing excessively enlarged (Figs $2 \mathrm{~A}$ and $\mathrm{B}$ ).

\section{Discussion}

We reported female fetal with type I CC based on prenatal MRI. Choledochal cysts are rare congenital anomalies, which appear as cystic or fusiform dilatation of the extra- or intrahepatic biliary duct. Choledochal cysts were classified according to the Todani modification of the Alonoso-Lej classification. Type I as solitary extrahepatic cyst, type II is an extrahepatic supraduodenal diverticulum, type III is intraduodenal cyst, type IV both extrahepatic and intrahepatic cysts, and type $V$ multiple intrahepatic cysts and often well known as Carolli's disease. Type I has been noted to be the most common. The female to male preponderance ranging from 4:1 to $3: 1 .^{1,2}$ Choledochal cysts are usually diagnosed in childhood, but with better resolution of US and MR diagnosed in utero is increased.

Ultrasound is still the modality of choice in prenatal screening, but it has limitation in the field of view and is operator dependent. Prenatal US revealed a cyst at hepatic hilum but GB cannot be evaluated. The patient was sent to radiology department with 

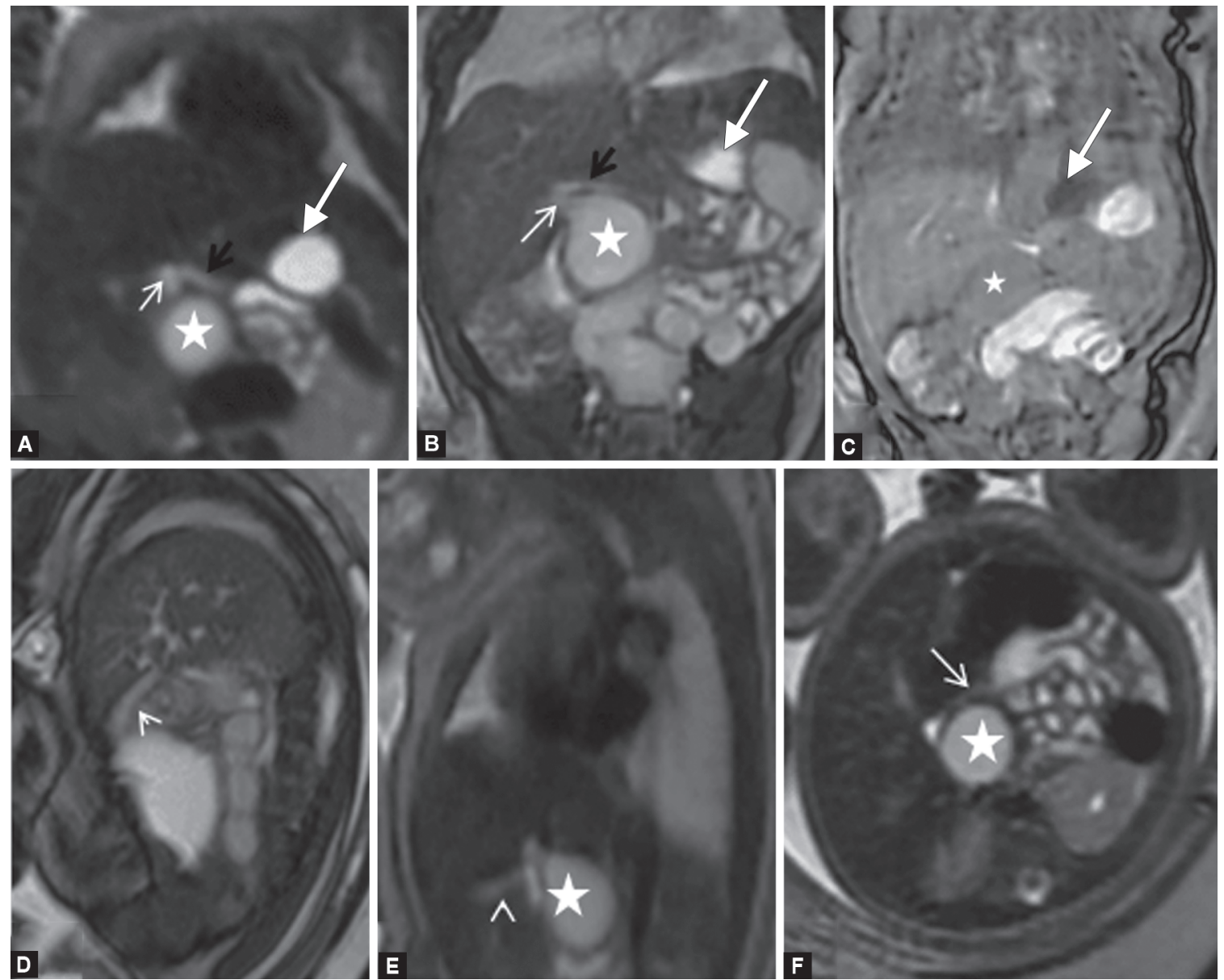

Figs 1 A to F: Coronal MRI choledochal cyst (asterisk) revealed hyperintense on T2 SSFSE (A) and T2 FIESTA (B), isointense to lung on T1-2DGRE (C), at cranial part of lesion showed tapered end structure (white arrow), intrahepatic biliary (black arrow) is not enlarged (A and B), the lesion separate with stomach (thick white arrow), large and small bowel. Sagittal T2 FIESTA (D) and T2 SSFSE (E) demonstrated normal gallbladder (head arrow) at inferior surface of the liver in right upper quadrant, cyst (asterisk) at extrahepatic, posterior orientation (E), on axial T2 SSFSE (F) revealed the cyst (asterisk) at posterior duodenum (white arrow) and separate with kidney
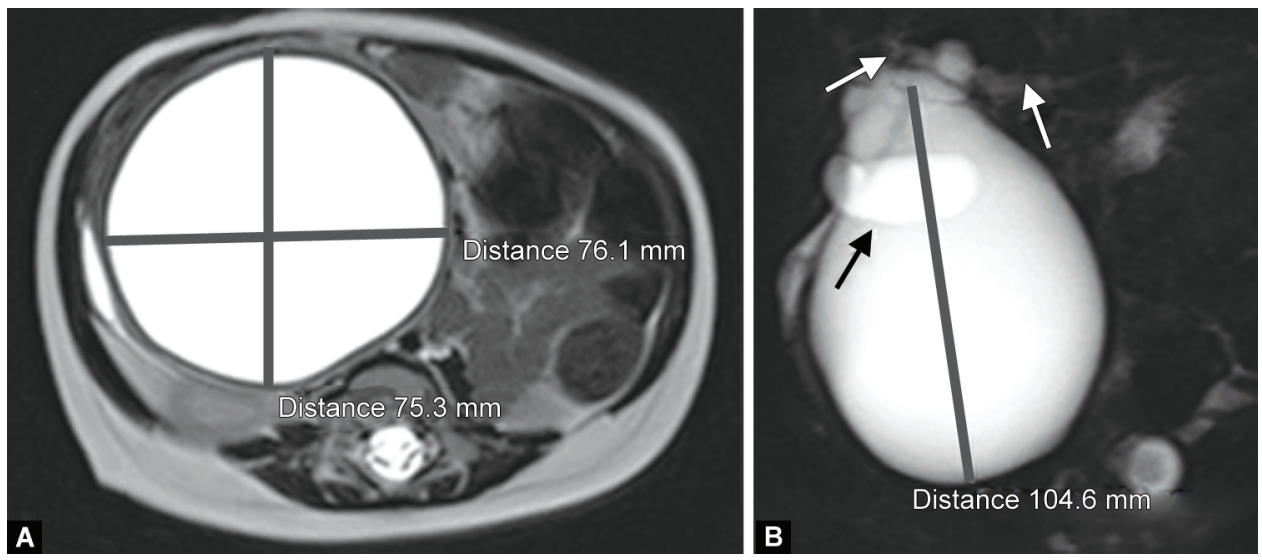

Figs $2 \mathrm{~A}$ and B: MRI images at 10 months of age, axial T2W (A), MRCP (B). MRI showed enlargement of the cyst size approximately $76.1 \times 75.3 \times$ $10.46 \mathrm{~mm}$ compressing right kidney (A), dilatation of left and right intrahepatic biliary (white arrow), hydrops gallbladder (arrow) (B)

possibility diagnosis of biliary atresia with cystic dilatation and CC as a differential diagnosis. Strengthening the diagnosis of this case is very important due to different timing and technique of surgery. Response to surgery in CBA is correlated with age at surgery; earlier surgical intervention results in improved outcome as determined by clearance of cholestasis. In contrast to CC may undergo delayed surgical intervention to allow patient growth. ${ }^{3}$

Fetal MRI can enhance US findings in CC, as reported by Chen et al. and Wong et al. ${ }^{4,5}$ On the other hand, according to MacKenzie fetal MRI has no additional information compare fetal 
US in diagnosing biliary atresia, both modalities are impossible to distinguish between CC and biliary atresia, so cannot be used as definitive diagnosis. ${ }^{6}$ In this case, fetal MRI shows the cyst at hepatic hilum, smooth wall, separated from the duodenum, hyperintense on $\mathrm{T} 2 \mathrm{~W}$, and isointense compared to lung on T1W. In younger fetus, intensity of the cyst is same with amniotic fluid on all sequences, but in older fetus as in this case the cyst became isointense to the lungs on T1W due to GB bile. ${ }^{7}$ Fetal MRI can excluded extrahepatic biliary atresia by normal finding size and shape of the GB. Visualization of fetal GB by US after 32 weeks of gestation is declined according to Hertzberg et al. ${ }^{8}$ Fetal MRI demonstrates connection between GB and cyst through cystic duct and also connection between cyst and biliary intrahepatic duct. At cranial part of the cyst fetal MRI shows of tapered shape, which has been explained by Wong et al. ${ }^{5}$ This sign may be specific to biliary tract anomaly but cannot exclude biliary atresia. Normal size and shape GB, depiction normal of intrahepatic ducts on T2FIESTA or T2 SSFSE sequences can exclude biliary atresia by fetal MRI. Nonvisualization of GB and visualization cyst specific to the biliary tract on fetal MRI are suggestive of biliary atresia according to Nori et al. ${ }^{9}$ Other possibilities such as ovarian cyst, mesenteric, adrenal, and renal cyst can be excluded because of different locations. Duodenal duplication can be excluded since less likely to have tapered ends. ${ }^{5}$ Based on these findings, CC type I was diagnosed.

Prenatal management of extrahepatic CC is conservative. In this case, the newborn baby has no icteric and cholic stool, so surgical intervention is not performed immediately. Follow-up US up to 4 months showed static size of the cyst. At 10 months of age, the baby has pale stool, abdomen distended with palpable mass, and progressive jaundice, but the baby looks calm. Follow-up MRI performed before surgery showed huge enlargement of cyst, hydrops GB, and intrahepatic biliary duct dilatation. The cyst was excised and a Roux-en-Y hepaticojejunostomy was performed. Histopathological result confirmed the diagnosis of CC. Postsurgery liver function and bilirubin were normal, total bilirubin $0.22 \mathrm{mg} /$ $\mathrm{dL}$ (normal <1.2), direct $0.09 \mathrm{mg} / \mathrm{dL}$ (normal <0.3). The baby was discharged with no jaundice and still normal thereafter.

\section{IMPRESSION}

Fetal MRI may enhance US findings in suspected cystic biliary atresia. Tapered end of the cyst could be specific sign of biliary tract anomaly. The relationship between the CC and cystic duct, GB, and intrahepatic duct can be seen clearly by fetal MRI. Normal size and shape GB, depiction of intrahepatic ducts on T2 FIESTA or T2 SSFSE sequences can exclude biliary atresia by fetal MRI.

\section{References}

1. Baison NG, Bons MM, Helton WS, et al. Choledochal cysts: similarities and differences between Asian and Western countries. World J Gastroenterol 2019;25(26):3334-3343. DOI: 10.3748/wjg.v25.i26. 3334.

2. Edil BH, Cameron J, Reddy S, et al. Choledochal cyst disease in children and adults: a 30 year single institution experience. J Am Coll Surg 2008;206(5):1000-1005. DOI: 10.1016/j.jamcollsurg.2007. 12.045.

3. Schooler GR, Alisha M. Cystic biliary atresia: a distinct clinical entity that may mimic choledochal cyst. Radiol Case Rep 2018;13(2):415-418. DOI: 10.1016/j.radcr.2018.01.025.

4. Chen $C P$, Cheng SJ, Chang TY, et al. Prenatal diagnosis of choledochal cyst using ultrasound and magnetic resonance imaging. Ultrasound Obstet Gynecol 2004;23(1):93-95. DOI: 10.1002/uog.912.

5. Wong AMC, Cheung YC, Liu YH, et al. Prenatal diagnosis of choledochal cyst using magnetic resonance imaging: a case report. World J Gastroenterol 2005;11(32):5082-5083. DOI: 10.3748/wjg.v11. i32.5082.

6. MacKenzie TC, Howell LJ, Flake AW, et al. The management of prenatally diagnosed choledochal cysts. J Pediatr Surg 2001;36(8):1241-1243. DOI: 10.1053/jpsu.2001.25784.

7. Brugger PC. MRI of the fetal abdomen Prayer D. Fetal MRI. Heidelberg: Springer; 2011. pp. 377-401.

8. Hertzberg BS, Kliewer MA, Maynor C, et al. Nonvisualization of the fetal gallbladder: frequency and prognostic importance. Radiology 1996;199(3):679-682. DOI: 10.1148/radiology.199.3.8637986.

9. Nori M, Venkateshwarlu J, Vijaysekhar, et al. Extrahepatic biliary atresia with choledochal cyst: prenatal MRI predicted and post natally confirmed: a case report. Indian J Radiol Imaging 2013;23(3):238-242. DOI: 10.4103/0971-3026.120278. 